\title{
Parallel Execution Profiles
}

\author{
Zachary Benavides \\ Univ. of California Riverside \\ benavidz@cs.ucr.edu
}

\author{
Rajiv Gupta \\ Univ. of California Riverside \\ gupta@cs.ucr.edu
}

\author{
Xiangyu Zhang \\ Purdue University \\ xyzhang@cs.purdue.edu
}

\begin{abstract}
Observing the relative behavior of an application's threads is critical to identifying performance bottlenecks and understanding their root causes. We present parallel execution profiles (PEPs), which capture the relative behavior of parallel threads in terms of the user selected code regions they execute. The user annotates the program to identify code regions of interest. The PEP divides the execution time of a multithreaded application into time intervals or a sequence of frames during which the code regions being executed in parallel by application threads remain the same. PEPs can be easily analyzed to compute execution times spent by the application in interesting behavior states. This helps user understand the severity of common performance problems such as excessive waiting on events by threads, threads contending for locks, and the presence of straggler threads.
\end{abstract}

\section{CCS Concepts}

-Computing methodologies $\rightarrow$ Parallel computing methodologies; Parallel programming languages;

\section{Keywords}

parallel behaviors; waiting; contention; load imbalance; stragglers; overhead; intrusion

\section{INTRODUCTION}

Understanding the runtime behavior of parallel programs is hard. Thus tools have been developed to understand parallel program behavior for performance debugging [1, 2, 4]. Existing works are aimed at specific metrics to identify performance bugs, e.g., normalized execution time, critical paths, slack etc. However, it is desirable to develop a single versatile framework that allows the user to capture a wide range of program behaviors and employ a variety of metrics to understand their causes. In addition, the framework should be lightweight and accurate. The instrumentation must be lightweight because its intrusive effects have the potential of altering the program's runtime behavior (e.g.,

Permission to make digital or hard copies of all or part of this work for personal or classroom use is granted without fee provided that copies are not made or distributed for profit or commercial advantage and that copies bear this notice and the full citation on the first page. Copyrights for components of this work owned by others than ACM must be honored. Abstracting with credit is permitted. To copy otherwise, or republish, to post on servers or to redistribute to lists, requires prior specific permission and/or a fee. Request permissions from permissions@ acm.org.

HPDC'16, May 31-June 04, 2016, Kyoto, Japan

(c) 2016 ACM. ISBN 978-1-4503-4314-5/16/05 . . \$15.00

DOI: http://dx.doi.org/10.1145/2907294.2907311 make contention appear or disappear). Sampling can be used to reduce runtime overhead [1]; however, it sacrifices the precision with which the runtime behavior is captured.

We present a framework for the collection and representation of runtime profiles of parallel executions that is versatile, accurate, and lightweight. Its key components are:

- Code Region Annotations. Our framework provides the user with an annotation mechanism using which code regions of interest are marked and named. This makes our system versatile as the user can select code regions of different types (e.g., code blocks, loops, functions, etc.) to match the needs for detecting interesting program behaviors (e.g., excessive waiting on events, locks with high contention, threads that are frequent stragglers).

- Parallel Execution Profile Representation. Code annotations guide the introduction of lightweight instrumentation via which per thread local timestamped event traces are collected while minimally perturbing the runtime behavior. Following program execution, the traces are converted into a novel representation called Parallel Execution Profiles (PEPs). A PEP divides the execution time of a multithreaded application into time intervals or a sequence of frames such that during each frame the code regions being executed in parallel by application threads remain the same. This representation is compact and precise. Interesting program behavior states involving multiple threads are characterized in terms of frames they generate.

- Analyzing PEPs. Since the PEP contains the activities of individual threads and their relative timing, a wide range of analyses to determine the frequencies and runtime durations of parallel behaviors can be determined. The user can build custom analyzers to identify performance bottlenecks and understand their root causes.

In our proposed approach, the user begins by developing a hypothesis for the cause of poor performance. Then, based upon this hypothesis, the user introduces annotations into the source code to identify code regions of interest. These annotations lead to instrumentation of the program that when executed produces timestamped traces for individual threads. The event traces are analyzed offline to generate a sequence of frames which describe what activities were performed by threads in parallel. Finally, the user constructs queries that reveal how often and for how long threads run in behavior states of interest, which reveals the absence or presence of the hypothesized performance problem. Moreover the above steps are applied iteratively till the program behavior is fully understood. 


\section{PARALLEL EXECUTION PROFILE}

In this section we first describe the per thread event trace that is generated when an annotated program is executed. Since event traces are thread local, they do not introduce any form of inter-thread synchronization, and thus do not perturb program behavior. Moreover, the overhead of trace collection is low because it uses lightweight instrumentation. Next we present a novel PEP representation that is derived offline, i.e. following program execution. The PEP representation consists of a series of frames. The local event trace of a thread tells us when the thread is executing a region of interest and when it is not. The frame sequence divides the application execution time into intervals where each frame captures the parallel behavior in terms of regions being executed by the threads in the interval.

Per Thread Event Trace represents the execution history of a single thread as a series of events and the times at which they took place. The event trace of thread $t$ that begins execution at time $s_{t}$, ends execution at time $e_{t}$, and along the way encounters region entry and exit events $e_{1} \cdots e_{n}$ at times $x_{1} \cdots x_{n}$ is denoted as follows:

$\left[t @ s_{t} \triangleright e_{1} @ x_{1} \triangleright e_{2} @ x_{2} \triangleright e_{3} @ x_{3} \triangleright \cdots \triangleright e_{n} @ x_{n} \triangleright\right] @ e_{t}$

The types of events captured by the event trace are:

- Thread creation and termination. A thread trace begins and ends with the events [tid and ] marking the creation of thread identified by tid and its termination.

- Region entry and exit. Intervening events are either region entry or region exit that are of the form:

$$
\begin{array}{ll}
- \text { Named only on entry } & \rightarrow \text { (rid .... }) ; \\
- \text { Named only on exit } & \rightarrow(\text {... rid }) ;
\end{array}
$$

Figure 5 visually shows the event traces of a pair of threads $t 1$ and $t 2$ executing regions of examples presented in Figures 1 through 4 in the preceding section. When a thread does not execute a region of interest, we use the name $\phi$.

Parallel Execution Profile (PEP) is represented in form of a sequence of frames where in each frame corresponds to the longest time interval over which, for all threads, the region being executed by each thread remains unchanged.

A frame is represented as follows where the time interval $[s, e)$ that it represents begins at $s$ (inclusive) and ends at $e$ (exclusive) and each $S\left(t i d_{i}\right)$ represents the state of thread tid $_{i}$ in terms of region(s) that it is executing.

$$
[s, e) \rightarrow\left[S\left(t i d_{1}\right)\right],\left[S\left(t i d_{2}\right)\right], \ldots\left[S\left(t i d_{n-1}\right)\right],\left[S\left(t i d_{n}\right)\right]
$$

If thread tid is in an unnested region, then $S(t i d)$ is one of the following: $\phi$ if thread tid is an unmarked region; and rid if thread tid is in a marked region. On the other hand if tid is nested in $n$ regions, then $S(t i d)$ has the following form: $S(t i d)=\left[\right.$ rid $_{1}$, rid $\left._{2}, \cdots r i d_{n}\right]$ where rid $_{1}$ is the outermost region and $r i d_{n}$ is the innermost region. The frames for executions of previously presented annotated code segments are shown in Figure 5.

Since PEPs give the global picture of the execution, unlike event traces that only describe individual threads, it is easy

\begin{tabular}{|c|c|c|c|c|c|c|}
\hline$[0],[0]$ & ${ }_{[}[0,1],[0,1]$ & ${ }_{[}^{[0],[0]}$ & $\left.\right|_{[} ^{1}[0,1],[0,1]$ & & ${ }_{[}^{1}[0],[0,1]$ & ${ }_{\mid}^{1}[0,1],[0]$ \\
\hline 0 & 1 & - & 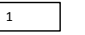 & $\circ \circ \circ$ & 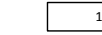 & 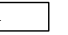 \\
\hline & i & & $\begin{array}{l}i \\
1\end{array}$ & & i & 1 \\
\hline 0 & 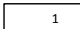 & & 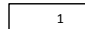 & 000 & 1 & \\
\hline
\end{tabular}
to characterize interesting behavior states in terms of the frames. For example, to detect a straggler thread we must (a) Execution of regions in Figure 1.

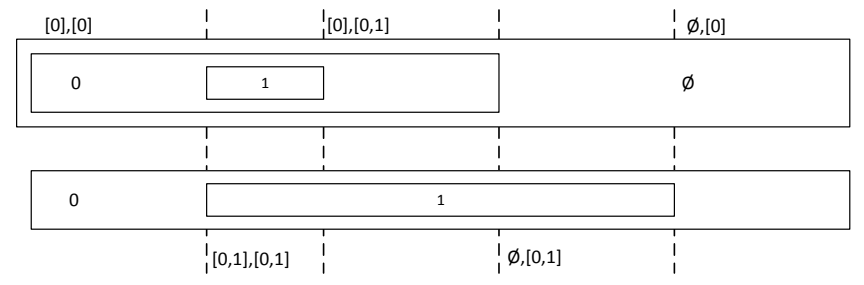

(b) Execution of regions in Figure 2.

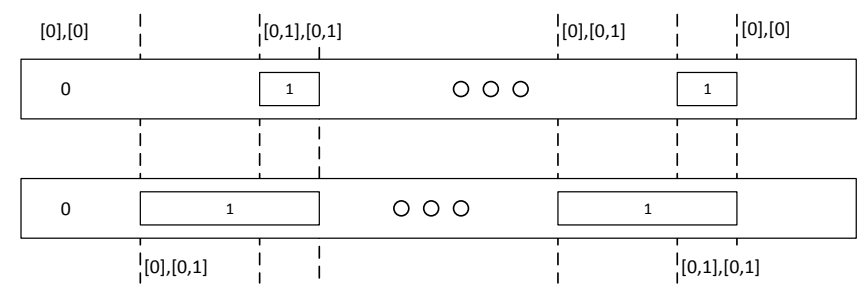

(c) Execution of regions in Figure 3 (left).

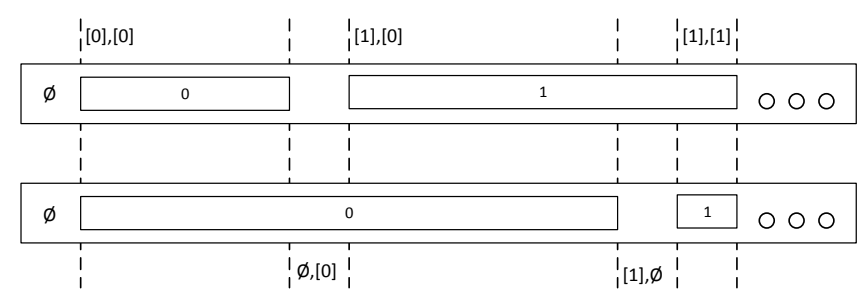

(d) Execution of regions in Figure 3 (right).

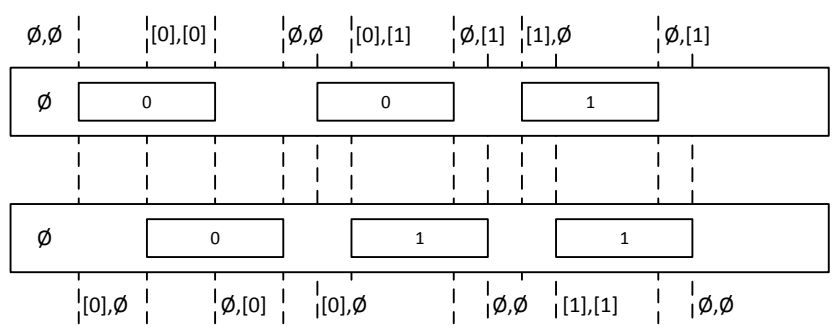

(e) Execution of regions in Figure 4.

Figure 5: Per Thread Event Traces Partitioned into a Sequence of Frames. 
search for frames of the following form where region $R_{1}$ represents the loop body preceding the barrier and region $R_{2}$ represents the barrier itself.

$$
\begin{gathered}
{\left[S\left(t i d_{1}\right)\right],\left[S\left(t i d_{2}\right)\right], \ldots\left[S\left(t i d_{n-1}\right)\right],\left[S\left(t i d_{n}\right)\right]} \\
\equiv \\
{\left[R_{2}\right],\left[R_{2}\right], \ldots\left[R_{2}\right],\left[R_{1}\right]}
\end{gathered}
$$

Note that threads $T_{1} \cdots T_{n-1}$ are waiting at the barrier while thread $T_{n}$ is executing the code preceding the barrier. The duration for which threads $T_{1} \cdots T_{n-1}$ wait at the barrier for thread $T_{n}$ is simply given by $x_{2}-x_{1}$. In the next section we will present additional examples that show how our query language allows user to easily analyze program behavior.

Performance Tuning Using PEPs. Next we illustrate the usage of PEPs to understand performance behaviors and tune performance for two commonly used parallel programming patterns involving lock contention and stragglers.

To illustrate the case of contention, we computed the total waiting time among application threads of the bodytrack benchmark from Parsec suite - the program was run with 16 threads and waiting was measured by annotating calls to pthread_cond_wait, pthread_barrier_wait, and pthread_mutex_lock. The contention was found to be quite high. In an attempt to alleviate this high contention, we reduced the number of threads by half, and measured the contention again. We found that the waiting time was reduced to $31.5 \%$ of original which translated into $2 \%$ reduction in overall execution time.

To illustrate the case of stragglers, let's examine the swaptions benchmark from Parsec. In an older version of Parsec, this benchmark was arranged so that work was not ideally distributed amongst the worker threads. When the native input set containing 128 work items is split amongst 13 threads, 12 of the threads are given 9 items, and the 13th thread is given 20 items. This leads to one of the application threads acting as a straggler. When we amend the work distribution so that 11 threads are given 10 items and 2 threads are given 9 items, we realize a speedup of $1.45 \mathrm{x}$.

\section{RELATED WORK}

A number of related works first introduce measures that assist in uncovering performance bottlenecks and then develop tools that efficiently collect runtime information for computing those metrics. [2] defines the notion of normalized processor time for a component as the ratio of the amount of processor time it consumes and the degree of parallelism. This metric is supported by the Quartz performance analysis tool. This approach essentially extends the gprof [5] style execution time profilers to handle multithreaded programs. [8] defines a metric called ParaShares as the normalized processor time with respect to weighted basic blocks. The key reason of using the above metrics for performance debugging is that the code which runs during periods of high parallelism has a smaller impact on overall execution time when optimized, than the code that runs during points of low parallelism.

Another popular technique for analyzing concurrent programs is based on the notion of a critical path that is defined to be the longest path through the program dependence graph that relates the activities of the program through their precedence relationships. In [7], the authors use hierarchical critical path analysis to build a tool for estimating the parallel speedup of each region in the program. [3] defines several new performance metrics based on critical path analysis with the intention of identifying performance problems, particularly load imbalance, in highly parallel systems. In [6] authors develop true zeroing which is a method for comparing performance metrics.

In [4] the authors present the Free Lunch profiler, a Java based tool for detecting lock contention. One of the most important contributions of this paper is the definition of what they call the free lunch metric, which is used to measure critical section pressure. Finally, there are also tools that make use of sampling and hardware performance counters to provide a highly scalable analysis [1].

\section{CONCLUDING REMARKS}

We presented a framework to capture and represent the runtime profile of a parallel application. Due to space limitations many important aspects of our work are not presented. First, the instrumentation for collecting event traces is lightweight and leads to modest execution time overhead of $0-7 \%$ for eight Parsec programs that were run on native inputs. Second, we support additional advanced annotations that allow capture of execution context of regions that is expressed in terms of dynamic names that are assigned to executed regions. Third, we have developed a rich query language that facilitates analysis of PEPs. The user can write complex queries that are implement to analyze PEPs automatically. Finally, we have carried out validation studies that demonstrate that our system captures runtime behavior with very high degree of precision.

\section{Acknowledgments}

This work is supported by NSF grants CCF-1524852 and CCF-1318103 to the University of California Riverside.

\section{REFERENCES}

[1] L. Adhianto, S. Banerjee, M. Fagan, M. Krentel, G. Marin, J. Mellor-Crummey, and N.R. Tallent. Hpctoolkit: Tools for performance analysis of optimized parallel programs. Concurrency and Computation: PESE, 22(6):685-701, 2010.

[2] T.E. Anderson and E.D. Lazowska. Quartz: A tool for tuning parallel program performance, In SIGMETRICS Perf. Eval. Review, 18(1):115-125, 1990.

[3] D. Bohme, F. Wolf, B.R. De Supinski, M. Schulz, and M. Geimer. Scalable critical-path based performance analysis. In IPDPS, pages 1330-1340, 2012.

[4] F. David, G. Thomas, J. Lawall, and G. Muller. Continuously measuring critical section pressure with the free-lunch profiler. In OOPSLA, 2014.

[5] S.L. Graham, P.B. Kessler, and M.K. Mckusick. Gprof: A call graph execution profiler. In $P L D I / C C$, pages 120-126, 1982.

[6] Jeffrey K Hollingsworth and Barton P Miller. Parallel program performance metrics: a comprison and validation. In ACM/IEEE Supercomputing, 1992.

[7] D. Jeon, S. Garcia, C. Louie, and M.B. Taylor. Kismet: parallel speedup estimates for serial programs. In OOPSLA, pages 519-536, 2011.

[8] M. Kambadur, K. Tang, and M.A. Kim. Parashares: Finding the important basic blocks in multithreaded programs. In Euro-Par, pages 75-86. Springer, 2014. 